\title{
An oral anticancer drug, TS-1, enabled a patient with advanced gastric cancer with Virchow's metastasis to receive curative resection
}

\author{
Takashi Imazawa ${ }^{1}$, Masakatsu Kinuta ${ }^{1}$, Hiroshi Yano $^{1}$, Shigeo Matsui ${ }^{1}$, Shinji Tamagaki ${ }^{1}$, Atsushi Yasue $^{1}$, \\ Kazuyuki Okada ${ }^{1}$, Toshiyuki Kanoh ${ }^{1}$, Takeshi Tono ${ }^{1}$, Yoshiaki NaKano $^{1}$, Shigeru OKamoto ${ }^{2}$, \\ and TAKuShi Monden ${ }^{1}$
}

${ }^{1}$ Department of Surgery, NTT West Osaka Hospital, 2-6-40 Karasugatsuji, Tennouji-ku, Osaka 543-8922, Japan

${ }^{2}$ Department of Pathology, NTT West Osaka Hospital, Osaka, Japan

\begin{abstract}
We encountered a patient with advanced gastric cancer, with Virchow's lymph node metastasis, who subsequently underwent curative resection after neoadjuvant chemotherapy with the newly developed oral anticancer drug, TS-1. The patient was a 67-year-old woman who had a type 2 tumor in the middle third of the stomach, and Virchow's lymph node metastasis, which was diagnosed by fine-needle aspiration cytology; she also had swollen paraaortic lymph nodes. Curative resection was considered impossible, and TS-1 (100 mg/day) was administered for 28 days in one course, mainly in the outpatient clinic. Although grade 2 stomatitis interrupted the therapy on day 21 of the second course and on day 7 of the third course, the type 2 tumor showed marked remission (partial response; PR) and the metastasis in the Virchow's and paraaortic lymph nodes had completely disappeared after the third course (complete response; CR). Eleven weeks after the completion of the TS-1 treatment, total gastric resection with D3 lymph node dissection was performed. Histopathological examination revealed tumor involvement only in the mucosal and submucosal layers of the stomach and the no. 4d lymph node. Most of the tumor was replaced with fibrosis with granulomatous change in the muscularis propria of the stomach and in the no. 3, no. 6, and no. 7 lymph nodes. This may be the first report of a patient with advanced gastric cancer with Virchow's lymph node metastasis who successfully received curative resection following neoadjuvant chemotherapy with a single oral anticancer drug.
\end{abstract}

Key words TS-1 · Virchow's lymph node metastasis · Gastric cancer · Gastrectomy · Neoadjuvant chemotherapy

\section{Introduction}

Gastric cancer with involvement of lymph nodes in the left supraclavicular fossa (Virchow's lymph node) is

Offprint requests to: T. Iwazawa

Received: August 7, 2001 / Accepted: January 28, 2002 considered to be stage IV, with distant metastasis (M1), according to the Japanese classification of gastric cancer [1], and is usually not an indication for surgery. The prognosis of unresectable stage IV gastric cancer is extremely poor, and several chemotherapy regimens have been introduced to attempt to prolong survival $[2,3]$ or to achieve downstaging, followed by curative resection $[4,5]$. However, to the best of our knowledge, few previous reports have documented chemotherapy that enables the curative resection of gastric cancer with metastasis to Virchow's lymph node, even though the response rate of recent combined chemotherapeutic modalities is $30 \%$ to $50 \%$. We encountered a patient with gastric cancer with Virchow's lymph node metastasis, who subsequently received curative resection following treatment with the newly developed oral anticancer drug, TS-1. There were no significant adverse reactions to the chemotherapy.

\section{Case report}

A 67-year old woman, who had complained of upper abdominal discomfort for 3 months, presented on June 6, 2000, with advanced gastric cancer, with swelling of Virchow's lymph nodes. Gastrointestinal fiberscopy (GIF) and upper gastrointestinal series (UGI) showed a type 2 tumor, i.e., ulcerated carcinomas with sharply demarcated and raised margins, on the greater curvature in the middle third of the stomach (Fig. 1A,C). A biopsy specimen showed poorly-to-moderately differentiated adenocarcinoma. Four swollen lymph nodes, up to $1.5 \mathrm{~cm}$ diameter, in the left supraclavicular area were considered to be metastasis to Virchow's lymph node, based on fine-needle aspiration cytology (Fig. 2). Abdominal computed tomography (CT) and ultrasound sonography (USG) showed swelling of several paraaortic lymph nodes (Fig. 3A; USG is not shown). Abdominal magnetic resonance imaging (MRI) showed 

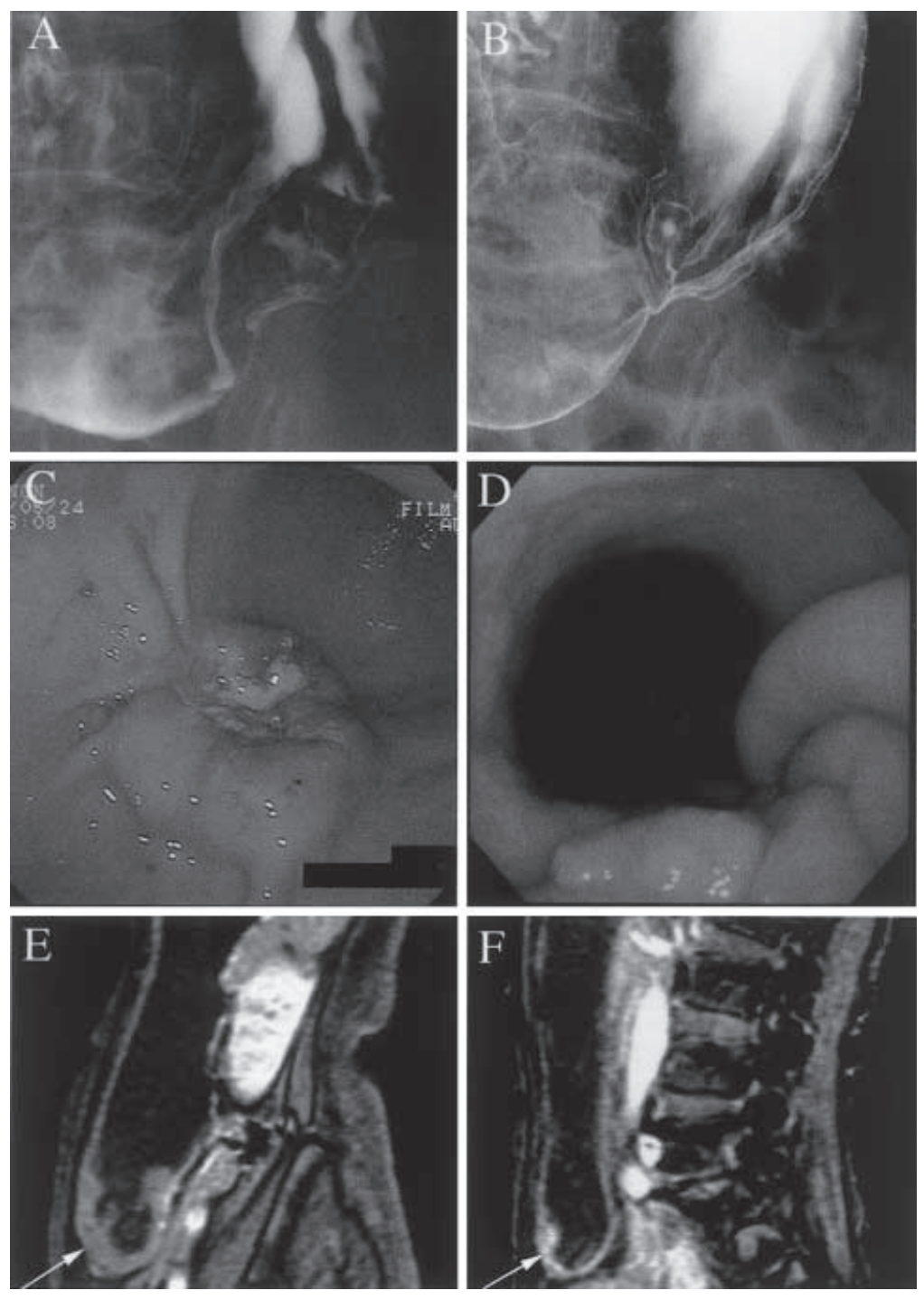

Fig. 1. A,B Upper gastrointestinal series shows a protruding lesion with a crater on the greater curvature of the middle third of the stomach before treatment (A); the crater and margin of the tumor were flattened after treatment (B). Evaluation of the response was partial response (PR). C,D Endoscopic findings show an irregularly shaped tumor with a crater before treatment $(\mathbf{C})$; the lesion had markedly regressed and flattened after treatment (D). E,F Abdominal dynamic magnetic resonance imaging (MRI) shows the thickness of gastric wall with high intensity (arrow) before treatment (E); the gastric wall became thinner (arrow) and the serosal surface became smooth after treatment $(\mathbf{F})$ thickening of the gastric wall and tumor invasion to the gastric serosa (Fig. 1E). The patient was given a diagnosis of stage IV (cT3, cN3, cP0, cH0, cM1) advanced gastric cancer with extensive lymph node metastasis, even though no distant metastasis to the liver, lung, bone, or peritoneum was diagnosed by CT, USG, or bone scintigraphy. The performance status of this patient was grade 0 , and laboratory examination results were within the normal ranges, except for a high level of carbohydrate antigen (CA)19-9 (283 U/ml). Because curative resection was impossible for this patient, chemotherapy, using TS-1, was started, on 22 June, 2000.

TS-1, $100 \mathrm{mg}$, was administered orally every day for 28 days, followed by a 14-day cessation as one course; the drug was administered mainly in the outpatient clinic. One course resulted in the complete disappearance of the swollen Virchow's lymph nodes, but slightly swollen paraaortic lymph nodes still remained on USG.
GIF showed marked reduction of the gastric tumor, but the presence of malignant cells was demonstrated by biopsy. During the second course of TS-1 administration, the patient had grade 2 stomatitis, so that the administration of TS-1 was interrupted on day 21. Although the third course of the treatment started after 35 days of rest, grade 2 stomatitis interrupted the administration again, on day 7. After this treatment, effectiveness was evaluated with UGI, GIF, CT, USG, and MRI. UGI and GIF revealed that the type 2 tumor had changed to an ulcer scar with fold convergence and a small elevated lesion (Figs. 1B,D; 4A), in which adenocarcinoma was proven by histological examination of the biopsy specimen. Dynamic abdominal MRI showed marked reduction of wall thickness after TS-1 administration and, finally, no serosal tumor invasion was demonstrated (Fig. 1F). Abdominal CT did not show any regional or paraaortic lymph node swelling (Fig. 3B), 

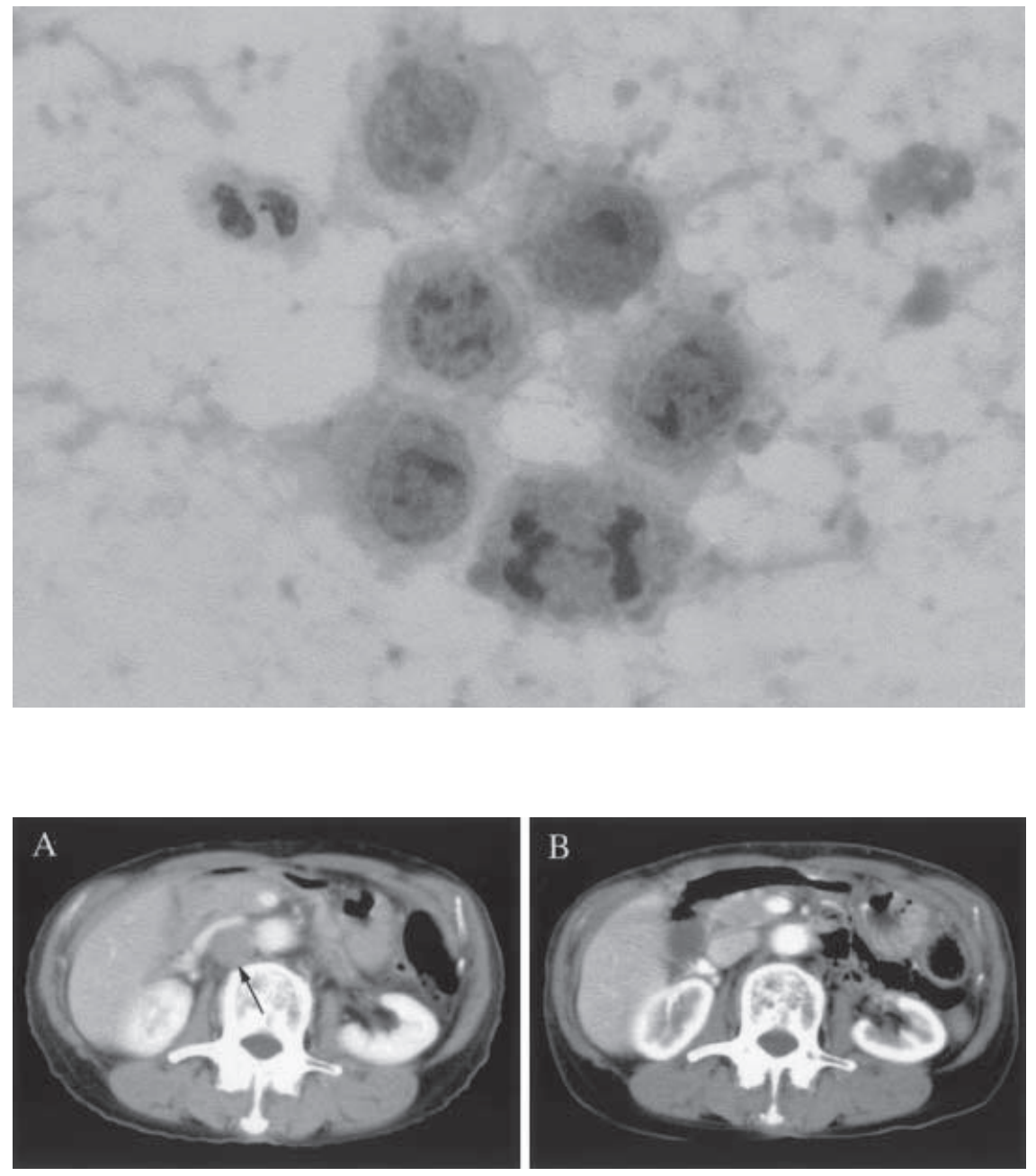

Fig. 2. Fine-needle aspiration cytology of Virchow's lymph node revealed a large number of adenocarcinoma cells with clear nuclei, a high nuclear/cytoplasmic $(\mathrm{N} / \mathrm{C})$ ratio, and mitosis. Papanicolaou stain, $\times 1000$

Fig. 3A,B. Enhanced abdominal computed tomography (CT) showed that lymph nodes around the abdominal aorta (no. 16a2) were swollen (arrow) before treatment (A); the swollen lymph nodes disappeared after treatment (B) abdominal USG showed a marked reduction of paraaortic lymph nodes (data not shown), and Virchow's lymph node was not palpable. The serum level of CA199 decreased to within the normal range, at $18 \mathrm{U} / \mathrm{ml}$. Therefore, we concluded that curative resection could be achieved.

Total gastrectomy with splenectomy and D3 lymph node dissection was performed on November 8, 2000, and no invasion to neighboring organs, no peritoneal dissemination, and no hepatic metastasis was recognized, and peritoneal cytology was negative. Histopathological examination showed submucosal invasion of a predominantly mucinous adenocarcinoma in a small elevated gastric lesion. Only xanthoma cells infiltrated the ulcer scar (Fig. 4A,B,C,D,E). Metastasis was found in only one lymph node, along the right gastroepiploic vessels (no. 4d), and no cancer cells were found in any other lymph nodes, including the paraaortic lymph nodes (no. 16a2 and 16b1). Necrosis and disappearance of the tumor, with granulomatous change, were observed in lymph nodes along the lesser curvature (no.
3), infrapyloric lymph nodes (no. 6), and lymph nodes along the left gastric artery (no. 7) (Fig. 4F). Eventually, the final stage was determined to be T1, N1, P0, CY0, $\mathrm{H} 0, \mathrm{Mx}$, and the curability of the surgical procedure was B (no residual tumors, but not evaluable as "curability A"). No signs of recurrence have been revealed by any examination 1 year after the surgery.

\section{Discussion}

Unresected gastric cancer has been treated by several regimens of combined chemotherapy. Some randomized control reports have documented that chemotherapy for gastric cancer patients with grade 0-2 performance status improved survival compared with best supportive care [6-8]. FAMTX (5-fluorouracil [5-FU] combined with Adriamycin [ADM] and Methotrexate [MTX]) is considered to be standard chemotherapy in Western countries $[9,10]$; however, it has not been accepted in Japan because of its severe toxicity. 

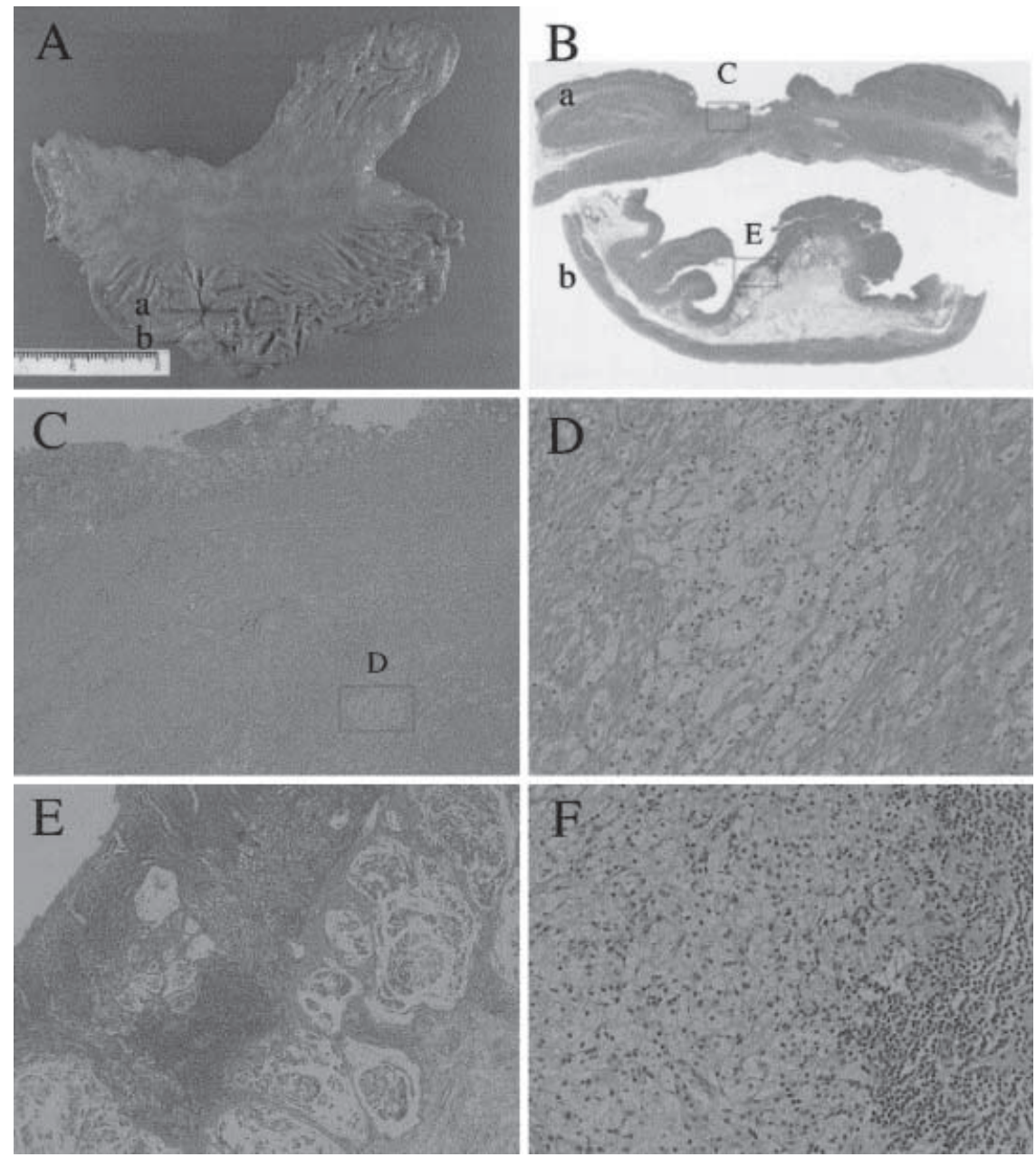

Fig. 4. A Macroscopic findings show an ulcer scar with fold convergence and neighboring small elevated lesion on the greater curvature in the middle third of the stomach B-F Histological findings showed that the gastric tumor was replaced by fibrosis with granulomatous changes and xanthoma cell infiltration in the ulcer scar $(\mathbf{B}, \mathbf{C}, \mathbf{D})$ and regional lymph nodes $(\mathbf{F})$. Dominantly mucinous adenocarcinoma with tubular adenocarcinoma invaded the submucosal layer of the elevated lesion $(\mathbf{B}, \mathbf{F})$. B H\&E, $\times 4$; C $\mathrm{H} \& \mathrm{E}, \times 40 ; \mathbf{D} H \& \mathrm{E}, \times 100 ; \mathbf{E} \mathrm{H} \& \mathrm{E}, \times 40$; F H\&E, $\times 100$
According to a Japanese Clinical Oncology Group (JCOG) study, FP (5-FU combined with cisplatin [CDDP]) therapy has better response rates than UFTM (UFT [tegafur, uracil] combined with mitomycin C [MMC]) and 5-FU alone [11], and continuous low-dose FP is used widely in Japan because of its high response rate, which is equal to that of the original FP protocol, with less toxicity [12]. The response rates of these chemotherapy regimens have improved to $30 \%-50 \%$, but no regimen yields better survival than continuous injection of 5-FU alone. Most patients suffer side effects, and often need long hospitalization for systemic chemotherapy, and thus, more effective anticancer drugs with milder toxicity are needed.

TS- 1 is a newly developed oral anticancer drug, which consists of tegafur, gimeracil, and oteracil potassium at a molecular ratio of $1: 0.4: 1$, based on the biochemical modulation of 5-FU [13]. Gimeracil competitively inhibits dihydropyrimidine dehydrogenase, is produced in various organs, including tumor tissues, and rapidly degrades 5-FU [14]. Oteracil potassium is an inhibitor of orotate phosphoribosyltransferase that catalyzes the phosphorylation of 5-FU, a process that is considered to be responsible for the toxic effects of 5-FU. Oteracil potassium is mainly distributed in the gastrointestinal tract after oral administration to rats, and induces amelioration of the gastrointestinal toxicity induced by 5 FU [15]. TS-1 induced a 53.6\% response rate for gastric cancer in an early phase II study [16] and a response rate of $49 \%$ in a late phase II study [17], with a $35.7 \%$ adverse reaction rate in the early phase II study and a $20 \%$ adverse reaction rate in the late phase II study. Not only is the response rate the highest for a single agent but also this oral anticancer drug does not require patient hospitalization, because of its mild toxicity. TS1 was more effective for lymph node metastasis (response rate of cervical lymph nodes, $68.4 \%$; abdominal lymph nodes, $49.2 \%$ ) than for the primary lesion $(32.6 \%)$, lung metastasis $(22.2 \%)$, or liver metastasis $(35.1 \%)$ in a phase II study. The present patient had lymph node metastasis at a distant site (Virchow's and paraaortic lymph nodes), but no metastasis to other organs, and no invasion to surrounding organs. Also, the performance status of the patient was grade 0 , and 
she had no problems ingesting food. Therefore, this patient was a good candidate for neoadjuvant chemotherapy with the oral anticancer drug, TS-1. In fact, the metastasis in Virchow's lymph node completely disappeared (CR) and the gastric tumor was reduced by more than $50 \%$ by TS-1; consequently, curative surgical resection could be performed. Histopathological examinations showed viable cancer cells to exist only in the mucosal and submucosal layer of stomach and in a group 1 lymph node, but not in any group 2 and group 3 lymph nodes, including paraaortic lymph nodes. More than two-thirds of the gastric tumor was replaced by fibrosis with granulomatous changes, in particular in the submucosal lesion and some regional lymph nodes. The response was classified as category grade 2 , moderate change, according to Japanese classification of gastric carcinoma. The final stage was T1 N1 P0 CY0 H0 Mx, and the level of curability of the surgery was B.

A few reports in Japan have documented the successful treatment of gastric cancer with Virchow's lymph node metastasis by chemotherapy. Ohyama et al. [18] reported a patient with advanced gastric cancer with Virchow's and paraaortic lymph node metastasis who completely responded to a four-drug combination chemotherapy. Pathological examination revealed no tumor cells in the primary lesion or in any dissected nodes, including Virchow's nodes, although recurrence developed 18 months after surgery [18]. Three reports have documented that Virchow's lymph node metastasis disappeared after low-dose FP chemotherapy, and that, consequently, gastrectomy could be performed, although paraaortic lymph node metastasis was histologically proven in all three patients [19-21]. Nakaguchi et al. [22] reported that Virchow's lymph node metastasis disappeared after treatment with an oral anticancer drug, 5'-deoxy-5-fluorouridine (5'-DFUR), and, consequently, distal gastrectomy was performed, but it was not curative surgery because of paraaortic lymph node metastasis. Therefore, the present report appears to be the first report of curative resection of advanced gastric cancer after the disappearance of Virchow's lymph node metastasis induced by neoadjuvant chemotherapy with a single oral anticancer drug. Complete response should be confirmed by longer observation. Although it is not yet known whether the overall survival of this patient has been improved, this case suggests that TS- 1 is an effective anticancer drug for advanced gastric cancer with extended lymph node metastasis. Moreover, this oral agent has the advantage of not requiring hospitalization for patients with good performance status, because of its mild toxicity.

Acknowledgment The authors gratefully acknowledge the assistance of Dr. Ogura, who provided UGI films before the treatment.

\section{References}

1. Japanese Gastric Cancer Association. Japanese classification of gastric carcinoma. 2nd English ed. Gastric Cancer 1998;1:10-24.

2. Preusser P, Wilke H, Achterrath W, Fink U, Lenaz L, Heinicke A, et al. Phase II study with the combination etoposide, doxorubicin, and cisplatin in advanced measurable gastric cancer. J Clin Oncol 1989;7:1310-7.

3. Boku N, Ohtsu A, Shimada Y, Shirao K, Seki S, Saito H, et al. Phase II study of a combination of irinotecan and cisplatin against metastatic gastric cancer. J Clin Oncol 1999;17:319-23.

4. Nakajima T, Ota K, Ishihara S, Oyama S, Nishi M, Ohashi Y, et al. Combined intensive chemotherapy and radical surgery for incurable gastric cancer. Ann Surg Oncol 1997;4:203-8.

5. Yonemura Y, Sawa T, Kinoshita K, Matsuki N, Fushida S, Tanaka S, et al. Neoadjuvant chemotherapy for high-grade advanced gastric cancer. World J Surg 1993;17:256-62.

6. Murad AM, Santiago FF, Petroianu A, Rocha PRS, Rodrigues MAG, Rausch M. Modified therapy with 5-fluorouracil, doxorubicin, and methotrexate in advanced gastric cancer. Cancer 1993; 72:37-41.

7. Glimelius B, Hoffman K, Haglund U, Nyren O, Sjoden PO. Initial or delayed chemotherapy with best supportive care in advanced gastric cancer. Ann Oncol 1994;5:189-90.

8. Pyrhonen S, Kuitunen T, Nyandoto P, Kouri M. Randomized comparison of fluorouracil, epidoxorubicin and methotrexate (FEMTX) plus supportive care with supportive care alone in patients with non-resectable gastric cancer. Br J Cancer 1995;71: 587-91.

9. Wils JA, Klein HO, Wagener DJT, Bleiberg H, Reis H, Korsten $\mathrm{F}$, et al. Sequential high-dose methotrexate and fluorouracil combined with doxorubicin - a step ahead in the treatment of advanced gastric cancer: a trial of the European Organization for Research and Treatment of Cancer Gastrointestinal Tract Cooperative Group. J Clin Oncol 1991;9:827-31.

10. Kelsen D, Atiq OT, Saltz L, Niedzwiecki D, Ginn D, Chapman D, et al. FAMTX versus etoposide, doxorubicin, and cisplatin: a random assignment trial in gastric cancer. J Clin Oncol 1992;10: $541-8$.

11. Shimada Y, Shirao K, Ohtsu A, Hyodo I, Saito H, Yamamichi N, et al. Phase III study of UFT + MMC versus 5-FU + CDDP versus 5-FU alone in patients with advanced gastric cancer: JCOG study 9205. Proc Am Soc Clin Oncol 1999;18:1043.

12. Chung YS, Yamashita Y, Nakata B, Nitta A, Inoue T, Hirayama $\mathrm{K}$, et al. Combination therapy of 5-FU and low dose CDDP for advanced and recurrent gastric cancer. Jpn J Cancer Chemother 1995;22:149-51.

13. Shirasaka T, Nakano K, Takechi T, Satake H, Uchida J, Fujioka A, et al. Antitumor activity of $1 \mathrm{M}$ tegafur- $0.4 \mathrm{M}$ 5-chloro-2,4dihydroxypyridine-1 $\mathrm{M}$ potassium oxonate (S-1) against human colon carcinoma orthotopically implanted into nude rats. Cancer Res 1996;56:2602-6.

14. Shirasaka T, Shimamoto Y, Ohshimo H, Yamaguchi M, Kato T, Yonekura K, et al. Development of a novel form of an oral 5fluorouracil derivative (S-1) directed to the potentiation of the tumor selective cytotoxicity of 5-fluorouracil by two biochemical modulators. Anticancer Drugs 1996;7:548-57.

15. Takechi T, Nakano K, Uchida J, Mita A, Toko K, Takeda S, et al. Antitumor activity and low intestinal toxicity of S-1, a new formulation of oral tegafur, in experimental tumor models in rats. Cancer Chemother Pharmacol 1997;39:205-11.

16. Sugimachi K, Maehara Y, Horikoshi N, Shimada Y, Sakata Y, Mitachi Y, et al. An early phase II study of oral S-1, a newly developed 5-fluorouracil derivative for treating patients with advanced and recurrent gastrointestinal cancers. Oncology 1999; 57:202-10.

17. Sakata Y, Ohtsu A, Horikoshi N, Sugimachi K, Mitachi Y, Taguchi T. Late phase II study of novel oral fluoropyrimidine 
anticancer drug S-1 (1 M Tegafur- $0.4 \mathrm{M}$ gimestat-1 M otastat potassium) in advanced gastric cancer patients. Eur J Cancer 1998;34:1715-20.

18. Ohyama S, Komatsu O, Nakajima T, Ohta K, Takahashi T, Yanagisawa A. Case report of pathological complete remission with FLEP therapy. In: Nakajima T, Yamaguchi T, editors. Multimodality therapy for gastric cancer. Berlin Heidelberg New York Tokyo: Springer-Verlag; 1999. pp. 104-7.

19. Umehara Y, Okubo T, Sano Y, Sakamoto R, Nakamura T, Tsuchiya $\mathrm{Y}$, et al. A case of advanced gastric remnant carcinoma with Virchow's metastasis treated with neoadjuvant chemotherapy (low dose CDDP + 5-FU) followed by surgical resection. Jpn J Cancer Chemother 1995;22:277-9.
20. Kajihara K, Ishikawa $\mathrm{H}$, Akama, F, Ninomiya $\mathrm{H}$, Shigeta $\mathrm{K}$, Sano I, et al. A case of advanced gastric cancer with Virchow's metastasis responding remarkably to combination chemotherapy of lowdose CDDP and 5-FU. Jpn J Cancer Chemother 1998;25:585-8.

21. Wada Y, Kamiya N, Asano S, Shinya F. A case of advanced gastric cancer with Virchow's and paraaortic lymph node metastases successfully resected after combined chemotherapy of low-dose CDDP and 5-FU. Jpn J Cancer Chemother 2001;28:7982.

22. Nakaguchi K, Nakano Y, Kitahara T, Onoe K, Nagamine H, Fukuda H. A resected case of gastric carcinoma with complete remission of Virchow's node metastasis by 5'-DFUR administration. Jpn J Cancer Chemother 1990;17:2101-4. 